\title{
Large Area EDS Mapping: Automated Collection of High Resolution Elemental Maps For Post Acquisition Analysis.
}

\author{
S. Burgess ${ }^{1}$, A. Hyde ${ }^{1}$, C. McCarthy ${ }^{1}$, Nigel Meeks ${ }^{3}$ \\ 1. Oxford Instruments Nanoanalysis, Halifax Road, High Wycombe, HP12 3SE, UK \\ 2. Department of Conservation \& Scientific Research, The British Museum, Great Russell Street, \\ London, WC1B 3DG
}

In microanalysis systems today (SEM/TEM) the limitations of high resolution imaging are constantly being enhanced, coupled with elemental analysis (EDS) means these systems can reveal significant information about small scale nanomaterials. However, for samples which are extremely large in size and contain a distribution of object sizes from micro to nano, analysing at high magnifications can misrepresent the chemical and structural composition of the entire sample. Furthermore, using low magnifications to overcome this issue produces maps which do not reveal the finer detail of the nanoscale features. This is particularly true in areas such as archaeology, geology, and materials science where micro- and nano-structure can have important implications.

At the British Museum in London, researchers are investigating the early Bronze Age smelting strategies used around the British Isles. EDS is used to analyse sections of large chalcopyrite ores, the products of smelting experiments, which have mixed copper-iron-sulphide chemistries. This work highlighted misunderstandings in the perception of the production of copper in prehistory. ${ }^{[1]}$ However, at the time, EDS experiments were limited to detectors with small senor sizes $\left(10 \mathrm{~mm}^{2}\right)$ and low X-ray count rates. This meant the analysis was time consuming and limited to small sections of the ore sample. Thorough investigation of the sample by SEM was required before beginning the lengthy task of collecting EDS maps. In the example used in this paper, the aim was to locate and understand the distribution of small copper prills within a partly smelted chalcopyrite ore matrix and to produce accurate values for the concentrations of the elements present.

X-ray Spectral map datacubes were collected from a grid pattern over the entire sample with AZtec Energy Large Area Mapping software and X-MaxN $80 \mathrm{~mm}^{2}$ SDD detector. The software automatically aligns and stitches individual datacubes together, to drastically increase the area of the ore which can be studied from one dataset, without losing resolution through low magnification settings, Fig 1. Using the $\mathrm{X}$-ray maps the distribution of copper prills across the entire sample is easily identified. Further analysis of the data in the cube was made to reconstruct the spectra from the copper prills. Although the data was collected at short process time and high count rate $(200,000 \mathrm{cps})$ suitable for fast X-ray mapping, the data quality is not affected, Fig 2. Any artefacts due to high count rate such as pulse-pile up are corrected, allowing accurate quantitative composition results to be obtained from the copper prills or other parts of the sample. Taking the data offline frees up valuable microscope time, and the image can be analysed further to study the distribution and composition of any of the structures which are present.

References:

[1] P. Craddock, N. Meeks and S.Timberlake, 2007, Metal and Mines. Studies in Archaeometallurgy (2007), p. 37-45. 

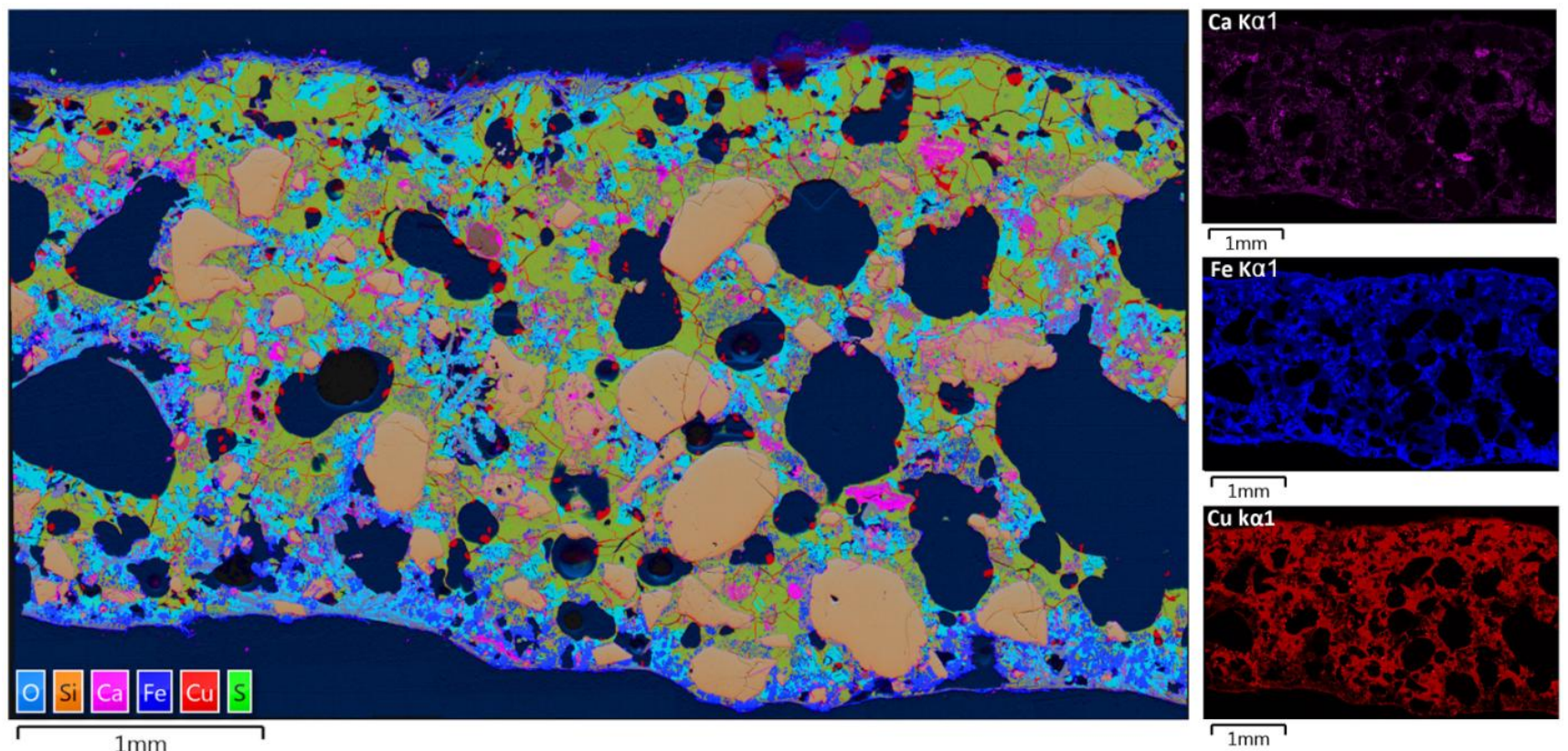

Figure 1. X-ray maps and 'Layered Image' calculated from the combined datacube. (The area analyzed is approximately 5 by $3 \mathrm{~mm}$ with a pixel result of 10,000 by 6,000 resulting in a pixel size of $0.5 \mu \mathrm{m}$ ).

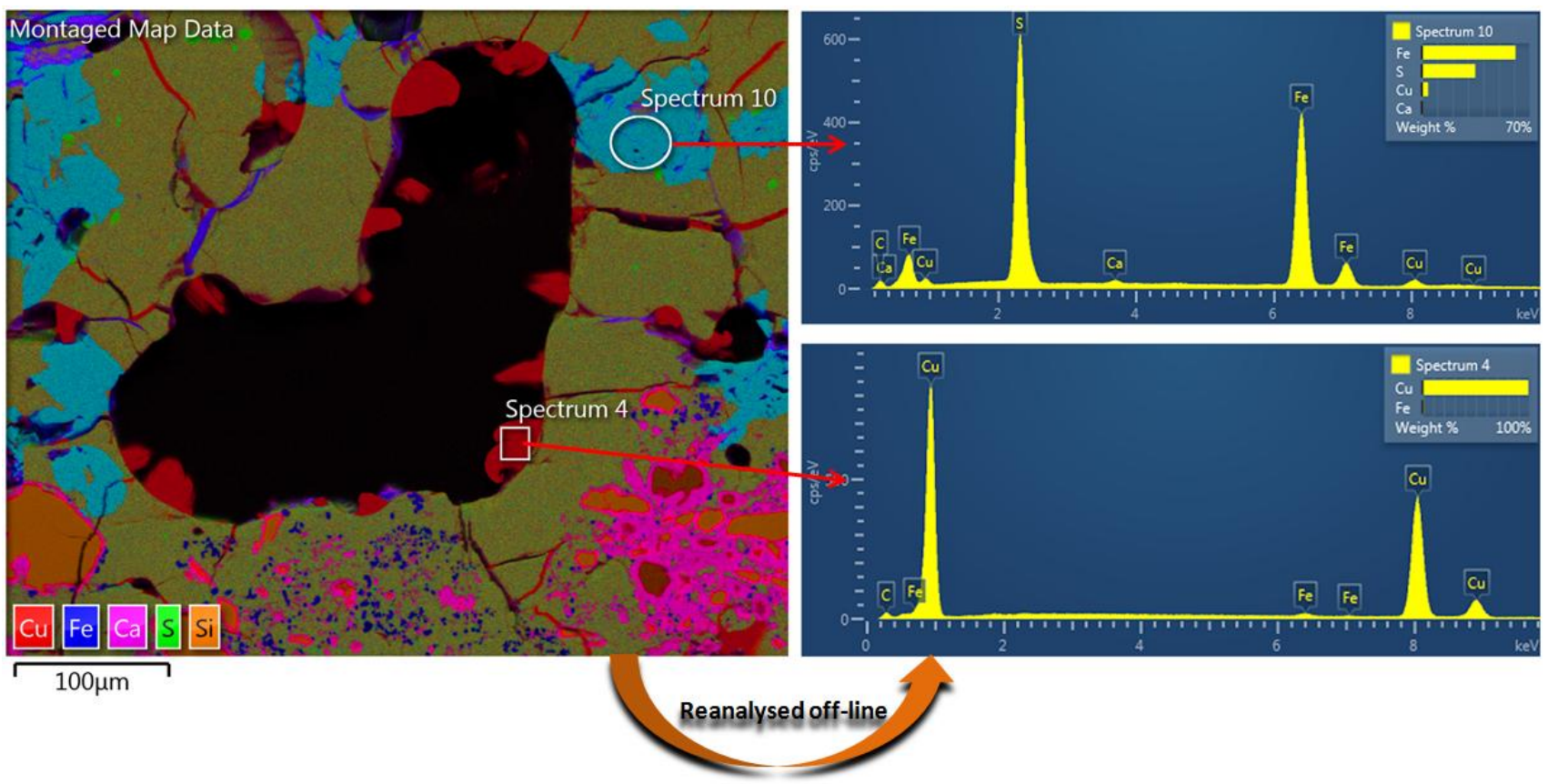

Figure 2. Layered Image showing the copper prills (red) in more detail. A spectrum reconstructed from one of the prills has been quantified to identify composition. 